\title{
Inhibition of Wind-Compressed Disk Inhibition in Optically Thick Winds
}

\author{
K.G. Gayley \\ University of Iowa, 203 Van Allen Hall, Iowa City, IA 52242
}

\begin{abstract}
There are three important effects in optically thin line-driven winds that have been shown to inhibit the equatorial compression inherent in the windcompressed disk model:

1) gravity darkening, which concentrates the driving flux over the poles,

2) stellar oblateness, which rotates the flux vectors poleward, and

3 ) nonisotropic opacity, which rotates the force vectors poleward.

The first two of these effects tend to yield fairly spherical winds, while the last one actually reverses the wind compression and should generate prolate winds. This poster argues that this third mechanism is not present for multiline scattering in Wolf-Rayet winds. Hence, it is argued that rotating optically thick linedriven winds should have a more spherical UV photosphere, especially for CAK $\alpha \cong 2 / 3$. However, owing to flux migration from polar to equatorial regions, such winds should have an even more prolate optical photosphere.
\end{abstract}

\section{Diffusive Flux in Optically Thick Winds}

Optically thick winds should inhibit flux migration on the scale of a stellar radius, thus encouraging flux to diffuse to regions of lower opacity near the equator. This in turn reduces the acceleration over the poles and enhances it near the equator. This velocity shear augments effective line opacity in the equatorial zone, leading to a more spherical UV photosphere, even as it reduces the density there and generates a more prolate optical photosphere.

The way diffusion affects the radiative force is easily determined by noting that a diffusive radiation field is isotropic to lowest order, and this in turn implies that the radiation pressure is simply $4 \pi J / 3$, where $J$ is the angle-averaged mean intensity. This gives for the radiative force per unit volume

$$
\rho \overrightarrow{\mathbf{g}}_{\text {rad }}=-\frac{4 \pi}{3} \nabla J .
$$

The diffusive radiative flux $\overrightarrow{\mathbf{F}}$, on the other hand, is

$$
\overrightarrow{\mathbf{F}}=-\int d \Omega \hat{\mathbf{n}} \frac{1}{\chi(\hat{\mathbf{n}})} \hat{\mathbf{n}} \cdot \nabla J
$$

which is complicated by the non-isotropic nature of the effective opacity $\chi$. Note from the above that if $J$ and $\chi$ are spherically symmetric, so are $\rho \overrightarrow{\mathbf{g}}_{\text {rad }}$ and $\overrightarrow{\mathbf{F}}$. Can the $J$ contours make a transition from being oblate in the interior of the rotating star, to being spherical in an optically thick wind? 


\section{A Schematic Model}

A "toy" model will now be described, which makes the following assumptions:

1) the surface flux aligns with, and is proportion to, the local effective gravity,

2) the mass flux in each wedge-shaped $\theta$ sector is assumed to stream radially,

3) gravity only weakly affects the wind velocity once the mass-loss rate is set, and

4) on the scale of a stellar radius, a transition occurs from von-Zeipel type flux to a spatially diffusive flux that is inversely proportional to the local opacity. To carry out this schematic solution, it is useful to replace the spherical geometry with a rectilinear coordinate system via the spatial transformation

$$
z=R_{p}\left(1-\frac{R_{p}}{r}\right) .
$$

Here $R_{p}$ is the polar radius of the static star, and all geometrically sensitive variables $y$ transform into

$$
\tilde{y}=\left(\frac{r}{R_{p}}\right)^{2} y .
$$

According to assumption (4) above, the flux makes a transition to obeying

$$
\tilde{F}(\theta) \propto \frac{1}{\tilde{\chi}(\theta)} .
$$

At this point, we have

$$
\tilde{a}(\theta) \propto \frac{\tilde{\chi}(\theta)}{\tilde{\rho}(\theta)} \tilde{F}(\theta) \propto \frac{1}{\tilde{\rho}(\theta)} \propto \frac{\tilde{v}(\theta)}{\tilde{g}_{\mathrm{eff}}(\theta)},
$$

where we have used the result from gravity darkening that the CAK mass flux is proportional to the effective gravity $g_{\text {eff }}(\theta)$ at the surface. Then since $\tilde{a}=$ $\tilde{v} d \tilde{v} / d z$, we have $\tilde{v}(\theta) \propto 1 / \tilde{g}_{\text {eff }}(\theta)$, and since the CAK line opacity obeys

$$
\tilde{\chi}(\theta) \propto \rho^{1-\alpha}\left(\frac{d \tilde{v}}{d z}\right)^{\alpha} \propto \tilde{g}_{\mathrm{eff}}(\theta)^{1-\alpha} \tilde{v}(\theta)^{2 \alpha-1} \propto \tilde{g}_{\mathrm{eff}}(\theta)^{2-3 \alpha},
$$

we get finally

$$
\tilde{\chi} \propto \tilde{g}_{o}(\theta)^{2-3 \alpha} .
$$

This has the interesting ramification that if $\alpha=2 / 3$, which is indeed a characteristic value, $\tilde{\chi}$ becomes independent of $\theta$. Thus for $\alpha=2 / 3$, the UV line photosphere is spherical, which is consistent with the assumed spherical flux. The asphericity in the density $\rho$, on the other hand, has been enhanced, and we obtain for $\alpha=2 / 3$

$$
\tilde{\rho}(\theta) \propto \frac{\tilde{g}_{\mathrm{eff}}(\theta)}{\tilde{v}(\theta)} \propto \tilde{g}_{\mathrm{eff}}(\theta)^{2} .
$$

